\title{
New Techniques for Composite Wing Manufacture
}

\author{
David Barton ${ }^{1}$, Hilary OCKendon ${ }^{2} \dagger$, Bernard Piette $^{3}$ and Robert \\ WHITTAKER $^{3}$ \\ ${ }^{1}$ University of Bristol \\ ${ }^{2}$ University of Oxford \\ ${ }^{3}$ University of Durham \\ 4 Univesity of East Anglia
}

(Communicated to MIIR on 27 June 2021)

Study Group: ESGI138. 16-20 July 2018, University of Bath and University of Bristol

Communicated by: C. Budd, University of Bath

Industrial Partner: Airbus

Presenter: Geoff Williams, Airbus

Team Members: David Barton, University of Bristol; Eduard Campillo-Funollett, University of Sussex; Mike Jeffrey, University of Bristol; Hilary Ockendon, University of Oxford; John Ockendon, University of Oxford; Bernard Piette, University of Durham; Emily Walsh, University of the West of England; Robert Whittaker, University of East Anglia.

Industrial Sector: Aerospace

Tools: Differential geometry, Chebychev nets

Key Words: Deformation of woven materials, Carbon fibre

MSC2020 Codes: 35, 53, 65

\section{Summary}

This report addresses the construction of carbon fibre wing boxes and the problems associated with using carbon fibre sheets rather than individual carbon fibre tapes. In the case that the wing boxes are developable surfaces the lay up of carbon fibre sheets is straightforward, since the fibres can follow the contours of the surface without any need for shearing or extension of the fibres. To further expand the potential design space for the wing boxes, this report investigates the lay up of sheets over non-developable surfaces where some shearing of the sheet is required to achieve the desired results. In this report, three analytical approaches are considered, driven by the results from numerical studies on different surface geometries. Each of the approaches offers insights as to the type of geometric perturbations achievable when constrained by a maximum shear angle.

$\dagger$ Corresponding author: hilary.ockendon@maths.ox.ac.uk 
New Techniques for Composite Wing Manufacture

\section{Report Authors and Contributors}

David Barton (University of Bristol), Hilary Ockendon (University of Oxford), Bernard Piette (University of Durham), Robert Whittaker (University of East Anglia)

\section{Contributors:}

David Barton (University of Bristol), Eduard Campillo-Funollet (University of Sussex), Mike Jeffrey (University of Bristol), Hilary Ockendon (University of Oxford), John Ockendon (University of Oxford), Bernard Piette (University of Durham), Emily Walsh (University of the West of England), Robert Wittaker (University of East Anglia)

\section{Contents}

1 Executive Summary $\quad 3$

2 Problem Description $\quad 4$

3 Model Problem $\quad 6$

4 Perturbation of the origami solution.

5 A Simple Special Solution 13

6 Alternative Analytic Treatment $\quad 14$

$\begin{array}{ll}7 \text { A Continuum Model } & 18\end{array}$

$\begin{array}{ll}8 & \text { Supporting materials }\end{array} 21$ 


\section{Executive Summary}

This report addresses the construction of carbon fibre wing boxes and the problems associated with using carbon fibre sheets rather than individual carbon fibre tapes. In the case that the wing boxes are developable surfaces the lay up of carbon fibre sheets is straightforward, since the fibres can follow the contours of the surface without any need for shearing or extension of the fibres. To further expand the potential design space for the wing boxes, this report investigates the lay up of sheets over non-developable surfaces where some shearing of the sheet is required to achieve the desired results. In this report, three analytical approaches are considered, driven by the results from numerical studies on different surface geometries. Each of the approaches offers insights as to the type of geometric perturbations achievable when constrained by a maximum shear angle. 


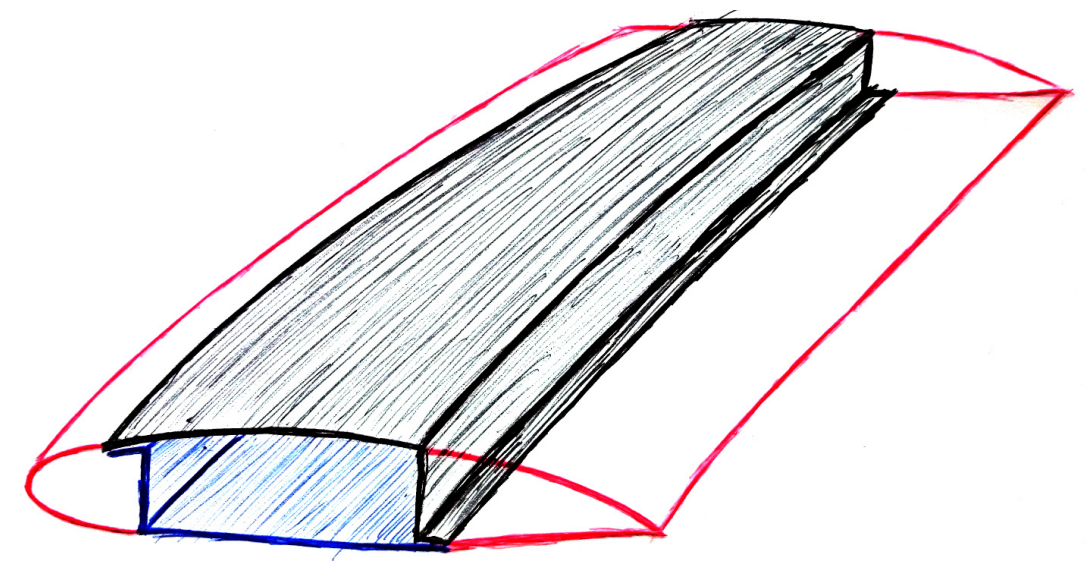

Figure 1: A sketch of a plane wing, showing the location of the wing box within in. The upper and lower components of the wing box are coloured black and blue, while the fore and aft extensions to the wing are shown in red.

\section{Problem Description}

Within the aerospace industry there is an ongoing trend to manufacture large parts of new aircraft using carbon fibre rather than aluminium/titanium as is more conventional. This trend presents challenges; there is considerable expertise available within the manufacturing community to produce a wide variety of complex geometries by machining aluminium - such expertise is not yet readily available for carbon-fibre parts which are constructed from flexible, but inelastic, fabric sheets.

Current wing box designs do not take into account the difficulties associated with carbonfibre manufacturing methods. In particular, they do not consider that they should be easily constructed from flexible sheets that cannot be stretched. Consequently, the manufacture of existing wing-box designs from carbon fibre sheets is time consuming and difficult.

The problem considered here is to characterise the design constraints for carbon-fibre wing boxes, in other words, to find which designs are straightforward to manufacture.

With carbon-fibre construction, the wing box is constructed as two separate components fastened together. Each part contains a wing surface (upper or lower) and a vertical spar that spans the cross-section of the wing. See figure 1.

We consider that the wing surface profile is given, with a set curvature both in the spanwise direction, and the fore-aft direction. The typical wing is $14 \mathrm{~m}$ meters in length and has a radius of curvature of the order of $65 \mathrm{~m}$. Moreover, the wing has a slope of $15^{\circ}$ near the aircraft, $7^{\circ}$ at a distance of $4 \mathrm{~m}$ from the aircraft and $3^{\circ}$ at the wing tip. 

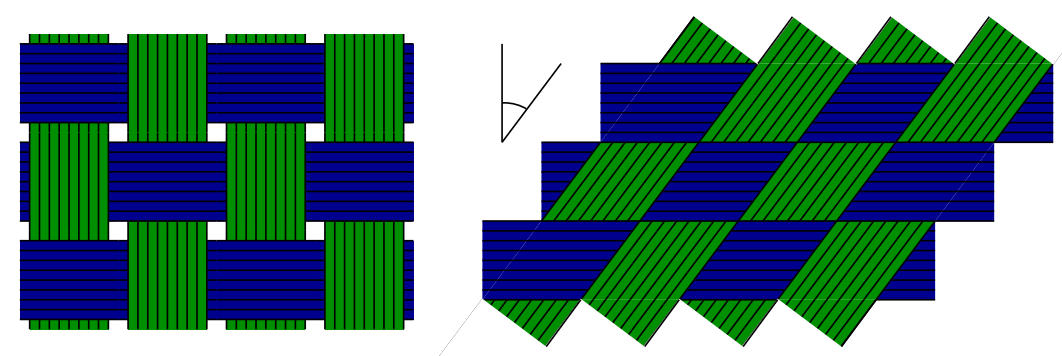

Figure 2: The simplest under-and-over carbon-fibre weave formed by ribbons comprising many individual fibres, initially crossing at right angles (left). Other weave patterns and initial fibre angles are also available. When subjected to simple shear, the gaps between the ribbons are reduced. The critical shear occurs when then ribbons come into contact (right).

A spar then joins to the wing cover, roughly at right-angles to the wing surface, with a height of the order of $1 \mathrm{~m}$.

The carbon fibre fabric comprises a woven mesh of individual fibres. Typically the fibres are grouped together to form flat ribbons, which are then woven in some pattern. These mesh fabrics do not stretch but can be sheared freely up to a critical shear angle (about $7{ }^{\circ}$ for the fabrics considered). The critical shear occurs when the ribbons come into contact, and the weave then prevents further shearing. See figure 2. In the construction process, several layers of carbon fibre fabric much be laid over a mould of the wing and spar shape. For structural strength, each single sheet should smoothly cover the wing and spar, without needing cuts or leaving wrinkles.

The Gaussian curvature of the wing surface is sufficiently small to allow the carbon-fibre sheets to be laid smoothly over the surface. Laying the carbon-fibre sheets over the edge and onto the spar presents greater difficulties. Airbus already appreciate that if two developable surfaces are joined by a so-called 'origami fold' (i.e. one in which the two surfaces could be flattened out into a single developable sheet) then the carbon fibre sheets can be applied without any problems. When attempting to construct wing box components with a deviation from such a fold, it has been found that is is difficult to get the sheets to lie smoothly over the surfaces without rucks appearing.

The design space of a wing box that comprises an origami fold is too small to be useful. Instead, the main problem is to determine if one can modify the curvature of the spar by some amount and still be able to cover the wing cover and spar smoothly with carbon fibre, only allowing shear deformation of the fabric and with a shear angle below a given bound.

The Study Group was therefore asked to investigate the laying of carbon fibre sheets over 
different edge geometries, with a particular focus on trying to quantify how much deviation is permitted from the ideal 'origami fold' given the critical shear angle of the fabric.

\section{Model Problem}

\subsection{Wing and Spar Geometry}

To simplify the problem we shall focus on the edge between the wing and the spar, and take the surfaces either side to be developable. Furthermore, we shall consider the special case where the generators are parallel within each surface, and that the two sets of generators are perpendicular to each other.

This leads to a natural coordinate system, in which the $x$ axis is parallel to the generators on the wing cover (roughly fore-aft) and the $z$ axis is parallel to the generators in the spar (roughly vertical). The $y$ axis is then oriented along the span of the wing. To describe the shape of the wing and spar we just need to specify how the curvature of each surface varies along the span of the wing.

We introduce two functions for the profiles of the two surfaces: $g_{w}(y)$ for the wing and $g_{s}(y)$ for the spar. The surface of the wing is then given by

$$
z=g_{w}(y) \text { for } x>g_{s}(y),
$$

while surface of the spar is given by

$$
x=g_{s}(y) \text { for } z<g_{w}(y) .
$$

An origami fold occurs precisely when the two surfaces have the same profiles, i.e.

$$
g_{w}(y)=g_{s}(y) .
$$

In this case, the intersection between the spar and the wing cover is a curve that lies on

a plane and the two surfaces can be covered by a single carbon-fibre sheet without any deformation.

We wish to investigate non-origami folds, i.e. $g_{w}(y) \neq g_{s}(y)$, and whether or not these can be covered smoothly by a carbon-fibre sheet subject to a given critical shear constraint.

\subsection{Carbon Fibre Model}

We focus on a single sheet of fabric consisting of two sets of inter-woven ribbons, which are initially at $90^{\circ}$ to each other. The carbon fibre weave size is of the order of a few 
millimetres, which is much smaller than the length scales of the wing cover and spar.

When it is deformed, the angle $\sigma$ between ribbons changes and we define the shear angle as the difference between that angle and a right angle:

$$
\theta_{\text {shear }}=\left|\frac{\pi}{2}-\sigma\right|
$$

We consider the fibre ribbons in the sheet as inextensible rods, hinged freely at the square lattice of cross-over points. The ribbons are able to bend both within the plane of the sheet (to allow for changes in the shear) and out of the plane (to allow for curvature in the surfaces and at the edge).

Because the scale of the fibres and the weave is much smaller than the dimensions of the wing, it is appropriate to use continuum approximation for the sheet if convenient.

For structural reasons, most of the carbon fibre sheets have fibres aligned along the length of the wing, so we shall focus on this case in this report. We shall generally assume that the fibre sheet is first laid undeformed on the developable wing surface and then folded around to cover the spar, with shearing on the spar introduced as necessary.

\subsection{Numerical Solution}

To provide insights into the resulting geometry of carbon fibre sheets draped over arbitrary surfaces, a numerical solution method was developed. The underlying assumptions embedded within the numerical method are as specified in sections 3.1 and 3.2 .

The solution process begins with fixing the positions of two non-parallel fibres on the specified surface. With these fibres as boundaries and for given weave size, the placement of all the remaining fibres within the carbon fibre sheet is entirely determined.

Nodes, representing the intersection of two fibres, are placed along each fibre with the distance between them being the weave size $\delta$. A complete grid of nodes is constructed starting at the intersection of the two initial fibres; two adjacent nodes are chosen and a point that is $\delta$ away from both nodes is found and a new node is placed there. See figure $\underline{3}$ for a schematic.

The problem of finding the location of a new node is a nonlinear root finding problem such that

$$
d\left(n_{1}, \tilde{n}\right)=\delta, \quad d\left(n_{2}, \tilde{n}\right)=\delta
$$

where $n_{1}$ and $n_{2}$ are the locations of two existing nodes, $\tilde{n}$ is the location of the new node, and $d(\cdot, \cdot)$ is the geodesic distance between two points on the specified surface. In the case 


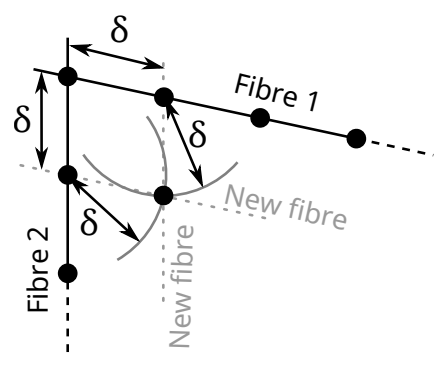

Figure 3: A schematic of the numerical construction of a grid of fibres.

where the surface is developable, the geodesic distance is obtained using the standard formula for arclength. A complication arises in the setting considered here due to the boundary between the surface of the wing and the surface of the spar; to obtain geodesic distances in this case, an optimisation problem is solved whereby the position that the fibre crosses the boundary is considered to be a parameter that can be varied to minimise the overall distance between the nodes.

For the numerical codes used in this report, please refer to section 8 .

\subsection{Qualitative Features of a Typical Solution}

A qualitative sketch of a typical solution is shown in figure $\underline{4}$. In this case we have used

$$
g_{w}(y) \approx 0.2 y^{2}, \quad g_{s}(y) \approx 0.4 y^{2} .
$$

The upper part shows the wing, and the lower the spar. In both cases the developable surfaces have been flattened out, so the horizontal coordinate is arc-length along each surface rather than $y$.

The carbon-fibre sheet is placed over the wing section without deformation, leading to a regular grid on the upper part of figure 4 . The layout on the spar in the lower part is then determined by fixing the left-most cross-wing ribbon (green) to be vertical, and by the fixed positions of both sets of ribbons that are imposed at the edge (black) from the solution on the wing.

There are two distinct regions of behaviour on the spar. Region I where along-wing ribbons (blue) originate from the left-hand boundary, and Region II where along-wing ribbons originate from the wing edge.

In Region I, the cross-wing ribbons (green) are vertical, and the along-wing ribbons (blue) all have the same shape, but are vertical translations of each other. The common shape of the along-wing ribbons is caused by vertical shifts in the cross-wing ribbons. There are two 
causes of these shifts. First the difference $g_{w}(y)-g_{s}(y)$ at the corner gives a vertical shift in the boundary condition at the top of the spar. Secondly, any deviation of the cross-wing ribbons from vertical in Region II causes an additional upward displacement of the sections lower down. We note that the gradient of the along-wing ribbons in Region I means that the cross-wing ribbons lie closer together towards the right-hand side of the spar.

In Region II there is shearing of the cross-wing ribbons, to match between the upper boundary at the wing and the lower boundary with Region I. At the upper boundary, the ribbons are displaced horizontally because the different gradients of the wing and spar result different arc-lengths in the $y$-direction along the two surfaces. In this example, the spar has larger gradient $\left(g_{s}^{\prime}(y)>g_{w}^{\prime}(y)\right)$ and so has a longer arc-length in the $y$ direction than the wing. Thus the spacing between the cross-wing ribbons is increased here at the top of the spar. At the lower boundary, the cross-wing ribbons are displaced horizontally because of the slope of the along-wing-ribbons in Region I. Thus the spacing between the cross-wing ribbons is decreased at the bottom of the spar.

We also note at this point that the solution in Region II is completely determined by the layout of the carbon-fibre sheet on the wing. In Region I, however, we have some flexibility, from the boundary condition imposed on the first cross-wing fibre at the left-hand side. In the example in figure 4, we could have placed a small negative horizontal shear at the lefthand side. This would have the effect of sheering the whole of Region II to the left. With an appropriate shear on the left-hand side, we could reduce the maximum shear found in Region II. This suggests it may be important how the sheet is laid down on the spar, and which end is fixed first.

The solution also points to a potential ill-posedness issue if an along-wing ribbon starts on the wing, goes over the edge onto the spar, and later crosses back over the edge and on to the wing. In this case, laying out the wing without deformation would impose boundary conditions at both ends of the section of the ribbon laying over the spar, leading to an illposed problem. One would need to include some shearing on the wing to accommodate this.

\section{Perturbation of the origami solution.}

In this section we consider a spar with a profile deviating from the origami solution. We describe the modified profile of the spar with

$$
g_{s}(y)=g_{w}(y)+f(y) .
$$

instead of (3), where $f(y)$ is the amount of deformation. This is illustrated on figures 5 and 6 . 


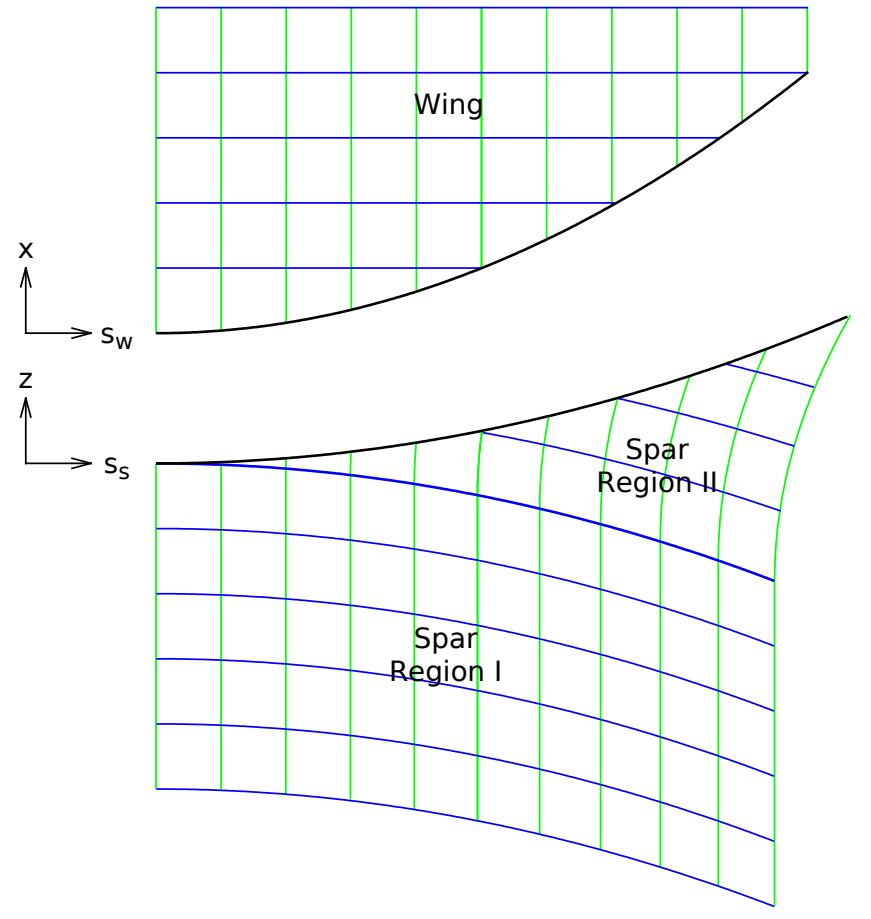

Figure 4: A qualitative sketch of a typical solution when laying a carbon fibre sheet over a wing and strut configuration that is not an origami solution. 


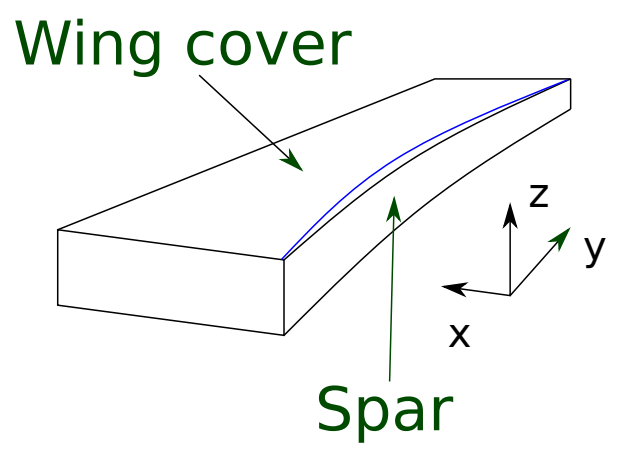

Figure 5: Wing with deformed spar. The blue curve illustrates how the spar can deviate from the origami solution profile

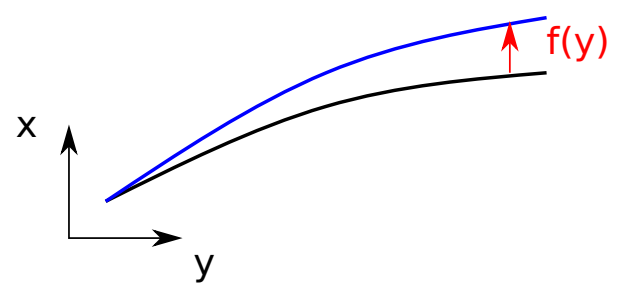

Figure 6: Spar profile: origami spar (black) and modified spar (blue).

We consider first the simplest origami solutions, i.e. without perturbation, where the coordinates of the carbon fibre nodes on the spar belonging to the same horizontal ribbon are all at the same level. We consider a single row at time and set $z=0$ for that row. Labelling the coordinates of the $i$ th junction along the row as $\left(x_{i}, y_{i}, z_{i}\right)$, we thus have

$$
z_{i}=0, \quad x_{i}=g_{s}\left(y_{i}\right)=g_{w}\left(y_{i}\right)=g_{i} .
$$

The $y_{i}$ are determined by the constraint that the distance between node is equal to the mesh size $\lambda$. By Pythagoras' theorem, we thus have

$$
\lambda^{2}=\left(x_{i+1}-x_{i}\right)^{2}+\left(y_{i+1}-y_{i}\right)^{2}+\left(z_{i+1}-z_{i}\right)^{2} .
$$

Introducing the horizontal spacing $l_{i}=y_{i+1}-y_{i}$, we have

$$
\lambda^{2}=\left(g_{i+1}-g_{i}\right)^{2}+l_{i}^{2} .
$$

Using the Taylor series of $g_{w}(y)$, and writing $g_{i}^{\prime}=g_{w}^{\prime}\left(y_{i}\right)$, we have

$$
g_{i+1} \approx g_{i}+g_{i}^{\prime} l_{i}
$$

and so $\lambda^{2}=\left(g_{i}^{\prime} l_{i}\right)^{2}+l_{i}^{2}$ leading to

$$
l_{i}=\frac{\lambda}{1+g_{i}^{\prime 2}}
$$

for the origami solution. 
If we modify the shape of the spar, with a perturbation $f(y)$, we then have the new coordinates of the $i$ th junction as $\left(\hat{x}_{i}, \hat{y}_{i}, \hat{z}_{i}\right)$ where

$$
\hat{x}_{i}=g_{w}\left(\hat{y}_{i}\right)+f_{i}\left(\hat{y}_{i}\right), \quad \hat{y}_{i}=y_{i}+\Delta y_{i}, \quad \hat{z}_{i}=-f_{i}\left(\hat{y}_{i}\right),
$$

where $\Delta y_{i}$ is the horizontal perturbation from the origami solution.

After the deformation, the distance between nodes must remain equal to the mesh size $\lambda$, so

$$
\begin{aligned}
\lambda^{2}= & \left(\hat{x}_{i+1}-\hat{x}_{i}\right)^{2}+\left(\hat{y}_{i+1}-\hat{y}_{i}\right)^{2}+\left(\hat{z}_{i+1}-\hat{z}_{i}\right)^{2} \\
= & \left(g_{i+1}-g_{i}+f_{i+1}-f_{i}+\left(g_{i+1}^{\prime}+f_{i+1}^{\prime}\right) \Delta y_{i+1}-\left(g_{i}^{\prime}+f_{i}^{\prime}\right) \Delta y_{i}\right)^{2} \\
& \quad+\left(y_{i+1}-y_{i}+\Delta y_{i+1}-\Delta y_{i}\right)^{2}+\left(f_{i+1}-f_{i}+f_{i+1}^{\prime} \Delta y_{i+1}-f_{i}^{\prime} \Delta y_{i}\right)^{2} .
\end{aligned}
$$

Defining

$$
S_{i}=\Delta y_{i+1}-\Delta y_{i}
$$

and using (9) we have

$$
\begin{aligned}
g_{i+1}-g_{i}+g_{i+1}^{\prime} \Delta y_{i+1}-g_{i}^{\prime} \Delta y_{i} & =g_{i}^{\prime} l_{i}+g_{i+1}^{\prime}\left(\Delta y_{i}+S_{i}\right)-g_{i}^{\prime} \Delta y_{i} \\
& =g_{i}^{\prime}\left(l_{i}+S_{i}\right)+g_{i}^{\prime \prime} l_{i}\left(\Delta y_{i}+S_{i}\right) .
\end{aligned}
$$

In the design of the wing, $g^{\prime}(y)$ is relatively small as $g^{\prime}(y) \approx 0.25$ for $15^{\circ}$. Moreover, the radius of curvature of the wing is much larger than the mesh size of the fibre and so $\lambda g^{\prime \prime}<<1$. As a result $g_{i}^{\prime \prime} l_{i}<<g_{i}^{\prime}$ and we can neglect the last term in (15). Similarly for $f$ we have

$$
f_{i+1}-f_{i}+f_{i+1}^{\prime} \Delta y_{i+1}-f_{i}^{\prime} \Delta y_{i} \approx f_{i}^{\prime}\left(l_{i}+S_{i}\right) .
$$

Combining (13), (15) and (16) we can write

$$
\lambda^{2} \approx\left(g_{i}^{\prime}\left(l_{i}+S_{i}\right)\right)^{2}+2\left(f_{i}^{\prime}\left(l_{i}+S_{i}\right)\right)^{2}+2 g_{i}^{\prime} f_{i}^{\prime}\left(l_{i}+S_{i}\right)^{2}+l_{i}^{2}+S_{i}^{2}+2 l_{i} s_{i}
$$

Then, using (10) we have

$$
\begin{aligned}
0 & =g_{i}^{\prime}\left(S_{i}^{2}+2 l_{i} S_{i}\right)+2\left(f_{i}^{\prime 2}+g_{i}^{\prime} f_{i}^{\prime}\right)\left(l_{i}^{2}+S_{i}^{2}+2 l_{i} S_{i}\right)+S_{i}^{2}+2 l_{i} S_{i} \\
& =\left(S_{i}^{2}+2 S_{i} l_{i}\right)\left(1+\left(g_{i}^{\prime}+f_{i}^{\prime}\right)^{2}+f_{i}^{\prime 2}\right)+2 l_{i}^{2}\left(f_{i}^{\prime 2}+f_{i}^{\prime} g_{i}^{\prime}\right)
\end{aligned}
$$

and so

$$
\begin{aligned}
S_{i} & =-l_{i}+l_{i} \sqrt{1-\frac{2\left(f_{i}^{\prime 2}+f_{i}^{\prime} g_{i}^{\prime}\right)}{1+\left(g_{i}^{\prime}+f_{i}^{\prime}\right)^{2}+f_{i}^{\prime 2}}} \\
& \approx-l_{i} \frac{f_{i}^{\prime 2}+f_{i}^{\prime} g_{i}^{\prime}}{1+\left(g_{i}^{\prime}+f_{i}^{\prime}\right)^{2}+f_{i}^{\prime 2}} .
\end{aligned}
$$

We thus see that the lateral distance between the vertical ribbons decrease by $S_{i}$ which is quadratic in the derivatives of $f$ and $g$ and can thus be neglected to first order approximation. 
This means that as the shape of the spar is modified, the first order effect is that the horizontal ribbons on the spar are simply shifted vertically by $f(y)$ and this induces a shear angle $\theta$ given by

$$
\begin{aligned}
\tan (\theta) & =\frac{z_{i+1}-z_{i}}{y_{i+1}-y_{i}} \\
& =\frac{f\left(y_{i+1}\right)-f\left(y_{i}\right)}{y_{i+1}-y_{i}} \\
& \approx \frac{\mathrm{d} f}{\mathrm{~d} y} .
\end{aligned}
$$

The shear angle $\theta_{\max }$ is then given by

$$
\theta_{\max }=\arctan \left(\max \left(\frac{\mathrm{d} f}{\mathrm{~d} y}\right)\right)
$$

This is confirmed by the numerical solutions, except that there is some extra shear of the fibre on the wing cover, but it is smaller than the shear on the spar. Hence our evaluation of the maximum shear is a good estimate.

\section{A Simple Special Solution}

If the spar is not curved but is vertical, there exists a very simple and special solution which can be constructed easily. For the spar we are considering, $g_{s}(y)=0$ everywhere. For this solution the carbon fibres on the wing cover are parallel and orthogonal to the edge of the (straight) spar. When they reach the spar, the strands must be pulled down vertically on the spar as illustrated on figure (7)

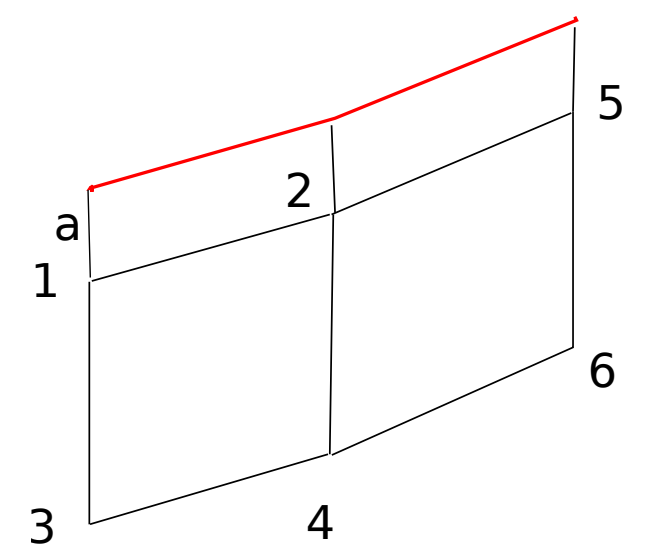

Figure 7: Flat spar solution: front view of the spar. The red line marks the edge between the spar and the wing cover.

If $\lambda$ is the length of a mesh and if the first parallel strand is at a distance $b$ from the spar edge, then the first nodes on the spar, 1,2 and 5, are at a distance $a=\lambda-b$ from the spar 
edge. We can then chose point 3 to be vertically below point 1 and at a distance $\lambda$. Point 4 will then be vertically below point 2 and at a distance $\lambda$ from it. This grantees that the distance between points 3 and 4 is also $\lambda$. Point 6 can the be positioned just below point 5 and we see that on the spar the nodes will be aligned on a curve parallel everywhere to the spar/wing interface.

We can then see that, as before, the maximum sheer is given by

$$
\theta_{\max }=\arctan \left(\max \left(\frac{\mathrm{d} f}{\mathrm{~d} y}\right)\right) \text {. }
$$

The main problem with this solution is that it appears to be highly sensitive to perturbations. It can be constructed numerically, but any small deviation leads to large shear on the wing cover or the spar.

\section{Alternative Analytic Treatment}

\subsection{Parallel ribbon segments}

We begin by making the observation that on a developable surface, the assumption about the ribbons being inextensible and pivoted at the crossing points means that each 'cell' bounded by four ribbon segments must be a parallelogram on the flattened surface. Therefore following a row or column of cells, the transverse ribbon segments must all be parallel to each other, i.e. have the same rotation.

In a manner reminiscent of characteristics, if the rotation angles of the segments are set at the boundaries, then rows and columns of cells can be followed in to the interior in order to determine the angles of the segments around each cell. This is depicted in figure $\underline{8}$. For each cell, the difference in the rotation angles of the two pairs of ribbon segments gives the shear angle of the cell.

\subsection{Separation of scales}

We make the assumption that $\left|g_{s}^{\prime \prime}\right|,\left|g_{w}^{\prime \prime}\right| \ll \lambda^{-1}$, which says that the variation in the gradient of the surfaces happens on a length scale much larger than the carbon-fibre mesh size. This means that locally, on the mesh scale, the edge between the wing and spar appears as a straight line to good approximation. The continuation of the mesh over the edge thus corresponds to a simple fold, and this allows us to make progress in what follows below.

Formally, we need to ensure that there is negligible change in the angle that the edge 


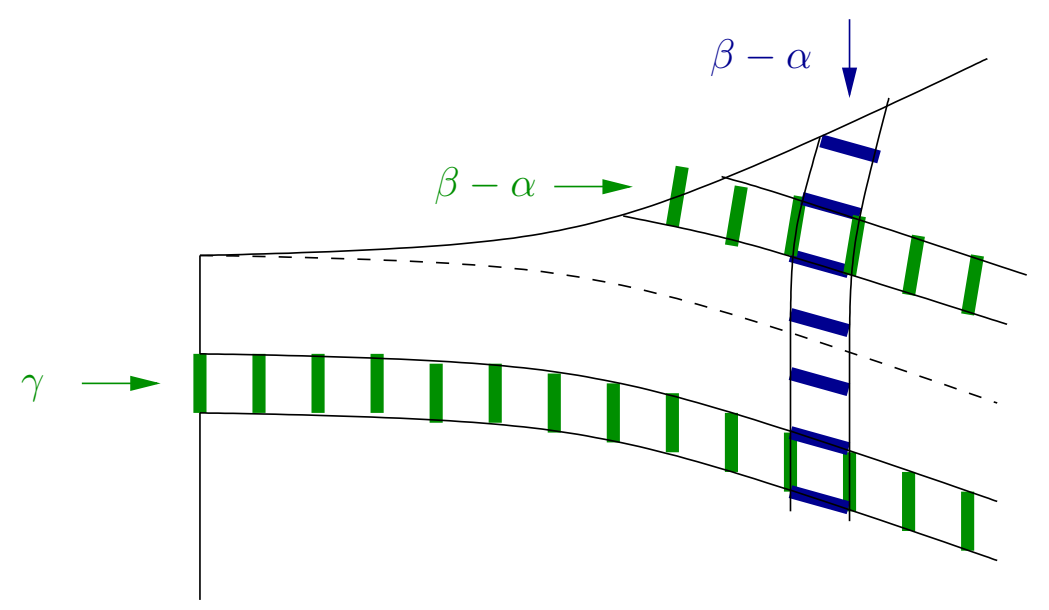

Figure 8: In each row or column of cells on the spar, the transverse ribbons segments must remain parallel. Therefore the rotation of the horizontal ribbon segments (blue) in each column of cells is set by the conditions at the wing edge, while the rotation of the vertical ribbon segments (green) in each row is set either by the left-hand boundary or by the conditions at the wing edge, depending on where the horizontal ribbon originated.

makes on the wing and spar surfaces between adjacent ribbons. Let $g_{\max }^{\prime \prime}$ be the largest absolute value of either second derivative, so that $\lambda g_{\max }^{\prime \prime} \ll 1$.

On the wing, cross-wise strands are at most a distance $\lambda$ apart in the $y$ coordinate. The change in the gradient of the edge on either surface is then at most the product of the longest possible distance, and the largest possible second-derivative: $\lambda g_{\max }^{\prime \prime}$ which we have assumed is small.

Shallow gradients in the wing and spar profiles can lead to span-wise ribbons being longer distances apart when they meet the edge. But we can still show that the deviation in the gradient between such ribbons much be small. Suppose that the difference between the maximum and minimum gradients between two span-wise ribbons is $D$. Then there is a point between them with a absolute gradient of at least $D / 2$. Then since the rate of change of the gradient is limited by $g_{\max }^{\prime \prime}$, there is a length

$$
\ell \geq \frac{D}{4 g_{\max }^{\prime \prime}}
$$

between the two ribbons, over which the modulus of the gradient is at least $D / 4$. Over this length, the $g$ changes by

$$
\Delta g \geq \ell \frac{D}{4} \geq \frac{D^{2}}{16 g_{\max }^{\prime \prime}}
$$

But we must have $\Delta g \leq \lambda$ for the edge to be within the two ribbons. Hence

$$
\frac{D^{2}}{16 g_{\max }^{\prime \prime}} \leq \lambda \quad \Rightarrow \quad D \leq 4\left(\lambda g_{\max }^{\prime \prime}\right)^{1 / 2} \ll 1
$$


The fact that the maximum deviation in the derivatives $g_{s}^{\prime}$ and $g_{w}^{\prime}$ is small between adjacent ribbons, means that the corresponding change in the angles $\alpha$ and $\beta$, as defined below in $\underline{26}$ and $\underline{27}$, must also be small. If these angles do not change much, then the edge is straight to good approximation.

\subsection{Local behaviour at the edge}

We introduce arc-length coordinates $s_{w}$ and $s_{s}$ in the $y$ direction on the wing and spar respectively. These are related to the $y$ coordinate in the usual way by

$$
\frac{\mathrm{d} s_{w}}{\mathrm{~d} y}=\left(1+{g_{w}^{\prime}}^{2}\right)^{1 / 2}, \quad \frac{\mathrm{d} s_{s}}{\mathrm{~d} y}=\left(1+{g_{s}^{\prime}}^{2}\right)^{1 / 2} .
$$

We now consider the mesh being folded over an approximately straight section of the edge in the neighbourhood of the point $\left(g_{s}(y), y, g_{w}(y)\right)$. The surfaces are shown in figure 9 in their flattened-out forms. We define $\alpha$ as the angle that the edge makes on the wing surface, relative to the $\left(s_{w}, z\right)$ coordinates. Similarly we define $\beta$ for the spar, relative to the $\left(s_{s}, x\right)$ coordinates. We then have

$$
\begin{aligned}
& \tan \alpha=\left.\frac{\mathrm{d} x}{\mathrm{~d} s_{w}}\right|_{\text {edge }}=\frac{\mathrm{d} g_{s}}{\mathrm{~d} y} \frac{\mathrm{d} y}{\mathrm{~d} s_{w}}=\frac{g_{s}^{\prime}}{\left(1+g_{w}^{\prime 2}\right)^{1 / 2}}, \\
& \tan \beta=\left.\frac{\mathrm{d} z}{\mathrm{~d} s_{s}}\right|_{\text {edge }}=\frac{\mathrm{d} g_{w}}{\mathrm{~d} y} \frac{\mathrm{d} y}{\mathrm{~d} s_{w}}=\frac{g_{w}^{\prime}}{\left(1+g_{s}^{\prime 2}\right)^{1 / 2}} .
\end{aligned}
$$

As shown in figure 9 , the mesh is offset by an angle $-\alpha$ relative to a line normal to the edge on the wing, and hence we have the same rotation relative to the normal line on the spar. This normal line is rotated from the vertical by the angle $\beta$, and hence the net rotation of the mesh on the spar is $\psi=\beta-\alpha$.

Thus immediately below the edge at the top of the spar, the rotation angle of both sets of ribbons is given by $\psi=\beta-\alpha$. This angle can be computed via

$$
\tan \psi=\tan (\beta-\alpha)=\frac{\tan \beta-\tan \alpha}{1+\tan \alpha \tan \beta}=\frac{g_{w}^{\prime}\left(1+g_{w}^{\prime}{ }^{2}\right)^{1 / 2}-g_{s}^{\prime}\left(1+g_{s}^{\prime 2}\right)^{1 / 2}}{\left(1+g_{w}^{\prime 2}\right)^{1 / 2}\left(1+g_{s}^{\prime 2}\right)^{1 / 2}+g_{w}^{\prime} g_{s}^{\prime}} .
$$

\subsection{Shear angles from ribbon rotations}

This rotation of the span-wise ribbons is propagated down the columns, while this rotation of the cross-wise ribbons is propagated along the rows, as shown in figure 8 . Additionally, cross-wise ribbons starting from the left-hand boundary of the spar (rather than originating on the wing) will have their rotation set by the boundary condition there. We denote the rotation angle of the vertical ribbons set there by $\gamma$. 


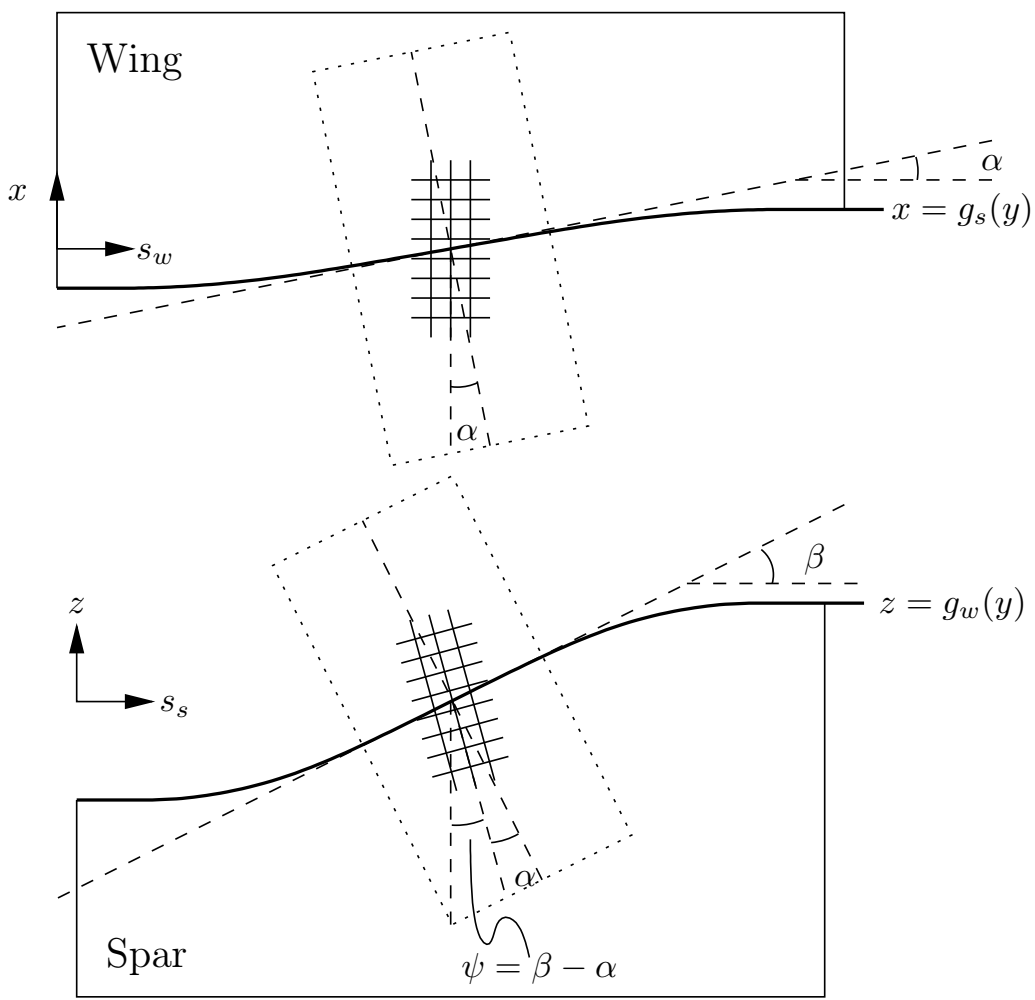

Figure 9: The rotation of the cells caused by a local straight fold at the wing-spar edge. Then the angle of the edge on the wing is $\alpha$ and the angle of the edge on the spar is $\beta$, then the rotation of the mesh due to the fold over the edge is $\beta-\alpha$. 
The shear angle experienced in each cell is then computed as the difference in the rotations of the span-wise and cross-wise ribbons that form its boundary. In the region reached by cross-wise ribbons originating on the wing, the maximum shear is given by the largest difference in $\psi$ values as $y$ varies.

$$
\theta_{\max , \mathrm{I}}=\max _{y, \hat{y}}|\psi(y)-\psi(\hat{y})|=\psi_{\max }-\psi_{\min },
$$

where $\psi_{\max }$ and $\psi_{\min }$ are the largest and smallest values of rotations $\psi$ from along the edge.

In the region reached by cross-wise ribbons originating on the boundary, the maximum shear is given by the largest difference between $\psi$ and $\gamma$ as $y$ varies.

$$
\theta_{\max , \mathrm{II}}=\max _{y}|\psi(y)-\gamma|
$$

This is minimised by taking $\gamma=\frac{1}{2}\left(\psi_{\max }+\psi_{\min }\right)$, giving

$$
\theta_{\max , \text { II }}=\frac{1}{2}\left(\psi_{\max }-\psi_{\min }\right) .
$$

Moreover for any $\gamma \in\left[\psi_{\min }, \psi_{\max }\right]$ we will have $\theta_{\max , \mathrm{II}} \leq \theta_{\max , \mathrm{I}}$, so (29) provides the effective constraint.

Insisting that $\theta_{\max , \mathrm{I}} \leq \theta^{*}$ is the maximum allowable shear angle in the carbon-fibre sheet,we obtain the condition

$$
\psi_{\max }-\psi_{\min } \leq \theta^{*}
$$

where $\psi(y)$ is computed from $g_{s}(y)$ and $g_{w}(y)$ using (28).

\section{A Continuum Model}

As mentioned in Section 2, the radius of curvature of the wing is much greater than mesh size and so it is reasonable to consider a continuum limit of infinitely small weave. The woven ribbons lie on a given surface and correspond to a continuous deformation of a 2parameter family of curves in this surface $z=z(x, y)$. If $u$ and $v$ are Lagrangian coordinates on $\mathbb{R}^{2}$ measured along the fibres, the surface can be described as $\mathbf{x}=(x, y, z(x, y))$ where $x$ and $y$ are functions of $u$ and $v$ which satisfy the equations

$$
\left|\frac{\partial \mathbf{x}}{\partial u}\right|=1, \quad\left|\frac{\partial \mathbf{x}}{\partial v}\right|=1
$$


These equations express the fact that the ribbons are inextensible and they can be written explicitly in the form

$$
\begin{aligned}
& \left(\frac{\partial x}{\partial u}\right)^{2}\left(1+\left(\frac{\partial z}{\partial x}\right)^{2}\right)+\left(\frac{\partial y}{\partial u}\right)^{2}\left(1+\left(\frac{\partial z}{\partial y}\right)^{2}\right)+2 \frac{\partial z}{\partial x} \frac{\partial x}{\partial u} \frac{\partial z}{\partial y} \frac{\partial y}{\partial u}=1 \\
& \left(\frac{\partial x}{\partial v}\right)^{2}\left(1+\left(\frac{\partial z}{\partial x}\right)^{2}\right)+\left(\frac{\partial y}{\partial v}\right)^{2}\left(1+\left(\frac{\partial z}{\partial y}\right)^{2}\right)+2 \frac{\partial z}{\partial x} \frac{\partial x}{\partial v} \frac{\partial z}{\partial y} \frac{\partial y}{\partial v}=1
\end{aligned}
$$

This model is the continuum limit of the discrete model described in Sections $\underline{4}$ and $\underline{6}$ and also in [1].

Equations (34) are nonlinear and do not fall under the usual classification of partial differential equations. However it can be shown that $u=$ constant and $v=$ constant are characteristics and thus prescribing $x$ on a fibre $u=$ constant and $y$ on a fibre $v=$ constant leads to a well-defined solution. We will now present some examples.

Since the relative rotation of the fibres is restricted by the geometry of the woven fabric it is necessary to add a constraint that the shear angle $\theta$ must be less than some limiting value $\theta_{\max }$ and this leads to the condition

$$
\cos \left(\frac{\pi}{2}-\theta\right)=\sin (\theta)=\left|\frac{\partial \mathbf{x}}{\partial u} \cdot \frac{\partial \mathbf{y}}{\partial v}\right| \leqslant \sin \left(\theta_{\max }\right) .
$$

In order to make analytical progress we will now consider the solution of the pair of equations (34) when the surface $z=z(x, y)$ only deviates from a plane by a small distance. This will allow us to solve the equations using a regular perturbation procedure and will give some useful insights. For most practical cases we will need to solve the problem numerically as is done below.

\subsection{A smooth convex surface}

We start by considering the woven material being draped over a smooth convex surface. We assume that the surface is given locally by

$$
z=-\sqrt{\epsilon}\left(a x^{2}+2 b x y+c y^{2}\right), \text { where } a c>b^{2}
$$

and $\epsilon$ is a small parameter. Then we can assume that the solution of (34) is a regular perturbation of the flat solution in which the fabric is unsheared and write

$$
z=0, x=u+\epsilon x_{1}, y=v+\epsilon y_{1} .
$$


In addition, we assume that $x=0$ on $u=0$ and $y=0$ on $v=0$ which determines the orientation of the fabric over the surface. The solution to $O(\epsilon)$ is then

$$
\begin{aligned}
& x=u-\frac{2}{3} \epsilon\left(a^{2} u^{3}+3 a b u^{2} v+3 b^{2} u v^{2}\right) \\
& y=v-\frac{2}{3} \epsilon\left(3 b^{2} u^{2} v+3 b c u v^{2}+c^{2} v^{3}\right)
\end{aligned}
$$

and the shear can be determined using $(\underline{35})$ as

$$
\sin (\theta)=2 \epsilon\left[a b u^{2}+2\left(a c-b^{2}\right) u v+b c v^{2}\right] .
$$

This analysis also holds in the limiting case when $a c=b^{2}$ and the surface is locally cylindrical. By taking $b=c=0$, we see that there will be no shear in the fabric when it is draped over the cylindrical shape $z=-\sqrt{\epsilon} a x^{2}$ as long as one of the weave directions lies along a generator of the cylinder. On the other hand, if we take $a c=b^{2}$ with $b \neq 0$ so that the Gaussian curvature is zero, but the fibres are not lined up with the generators of the cylinder, then the material will be subject to small shear as it falls over the cylinder.

The canonical form for an origami surface with zero Gaussian curvature (for example a cone) can be written locally as

$$
z=\sqrt{\epsilon}\left(k x+c y^{2}\right)
$$

and if we lay the fabric over the surface with a fibre along the $x$ axis, an analysis similar to that used above for the convex surface leads to the shear being given by $\sin \theta=2 \epsilon c k v$. Thus we see that the only surface which can be covered with no shear at all is a cylinder with one family of fibres lined up along its generators.

\subsection{A nearly straight wing edge}

We now consider the problem of covering the edge of a wing which was described in Section 3 . We take the surface of the wing to be

$$
\begin{array}{rll}
z=0 & \text { if } & x<\epsilon f(y) \\
z=-m(x-\epsilon f(y)) & \text { if } & x>\epsilon f(y) .
\end{array}
$$

where again $\epsilon$ is a small parameter. This takes the spar to be a plane of slope $m$ but by letting $m \rightarrow \infty$ we will be able to allow it to be perpendicular to the wing surface. We assume that when $\epsilon=0$ the fabric is spread over the two plane surfaces without any shear and oriented with one of the fibre directions along the $y$-axis. Then we can write

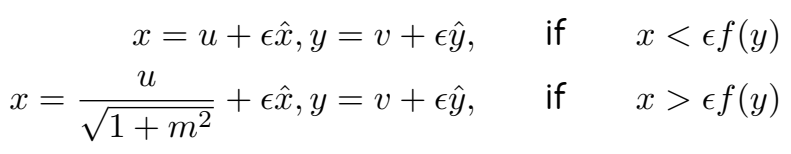


and take as boundary conditions $x=-1$ on $u=-1$ and $y=0$ on $v=0$. If we substitute these expressions into (34) and make $x$ and $y$ continuous across $z=-m(x-\epsilon f(y))$, then the solution, correct to the first order in $\epsilon$, is only affected in $x>\epsilon f(y)$ where it is

$$
\begin{aligned}
x & =\frac{u}{\sqrt{1+m^{2}}}+\epsilon f(v)\left(1-\frac{1}{\sqrt{1+m^{2}}}\right) \\
y & =v .
\end{aligned}
$$

From this solution we find that the shear angle is given by

$$
\sin (\theta)=\epsilon\left|f^{\prime}(y)\right|\left[1-\frac{1}{\sqrt{1+m^{2}}}\right] .
$$

Taking the limit $m \rightarrow \infty$, which corresponds to the two original planes being perpendicular, the shear angle is given by $\epsilon\left|f^{\prime}(y)\right|$ as found in Section 4. Thus, to avoid a shear of more than $\theta_{\max }$, we need to make sure that the perturbation to the wing edge is such that $\epsilon\left|f^{\prime}(y)\right|<$ $\sin \left(\theta_{\max }\right)$ for all $y$.

\subsection{Covering the corner of a cube}

There was some interest in whether it was possible to cover the corner of a cube without any wrinkling of the material. Using this model and assuming there is uniform shear in each quadrant of the $u v$ - plane it is possible to cover any pyramid by putting the origin at the vertex of the pyramid. The total shear needed to cover the corner of a cube is $\pi / 2$ since it is neccessary to reduce the angle $2 \pi$ on the flat unsheared surface to cover the 3 right angles at the corner. This means that the angle between the two families of fibres in each quadrant need to be sheared by $\pi / 8$.

\section{Supporting materials}

A ZIP file containing the source code for the numerical simulations performed is embedded within this PDF file. See the attachments tab within your PDF viewer to extract it. The source code is compatible with Julia vl.x, an open source programming language for technical computing. 
New Techniques for Composite Wing Manufacture

\section{References}

[1] S.B.Sharma and M.P.F.Sutcliffe, Draping of woven fabrics; Progressive drape model. Plastics, Rubber and Composites (2003) 32, 57-64. 